\title{
Scan-rescan reproducibility of quantitative assessment of inflammatory carotid atherosclerotic plaque using dynamic contrast-enhanced 3T CMR in a multi-center study
}

\author{
Huijun Chen ${ }^{1,2}$, Jie Sun², William S Kerwin², Niranjan Balu², Moni B Neradilek ${ }^{3}$ Daniel S Hippe², Daniel Isquith",
} Yunjing Xue ${ }^{2}$, Kiyofumi Yamada², Suzanne Peck ${ }^{4}$, Chun Yuan ${ }^{1,2}$, Kevin D O'Brien ${ }^{4}$ and Xue-Qiao Zhao ${ }^{4 *}$

\begin{abstract}
Background: The aim of this study is to investigate the inter-scan reproducibility of kinetic parameters in atherosclerotic plaque using dynamic contrast-enhanced (DCE) cardiovascular magnetic resonance (CMR) in a multi-center setting at 3T.

Methods: Carotid arteries of 51 subjects from 15 sites were scanned twice within two weeks on 3T scanners using a previously described DCE-CMR protocol. Imaging data with protocol compliance and sufficient image quality were analyzed to generate kinetic parameters of vessel wall, expressed as transfer constant $\left(K^{\text {trans }}\right)$ and plasma volume $\left(v_{p}\right)$. The inter-scan reproducibility was evaluated using intra-class correlation coefficient (ICC) and coefficient of variation (CV). Power analysis was carried out to provide sample size estimations for future prospective study.

Results: Ten (19.6\%) subjects were found to suffer from protocol violation, and another 6 (11.8\%) had poor image quality $(n=6)$ in at least one scan. In the $35(68.6 \%)$ subjects with complete data, the ICCs of $K^{\text {trans }}$ and $v_{p}$ were 0.65 and 0.28 , respectively. The CVs were $25 \%$ and $62 \%$, respectively. The ICC and CV for $v_{p}$ improved to 0.73 and $28 \%$ in larger lesions with analyzed area larger than $25 \mathrm{~mm}^{2}$. Power analysis based on the measured CV showed that 50 subjects per arm are sufficient to detect a $20 \%$ difference in change of $K^{\text {trans }}$ over time between treatment arms with $80 \%$ power without consideration of the dropout rate.

Conclusion: The result of this study indicates that quantitative measurement from DCE-CMR is feasible to detect changes with a relatively modest sample size in a prospective multi-center study despite the limitations. The relative high dropout rate suggested the critical needs for intensive operator training, optimized imaging protocol, and strict quality control in future studies.
\end{abstract}

Keywords: Carotid artery, Atherosclerosis, Reproducibility, Dynamic contrast-enhanced cardiovascular magnetic resonance

\section{Background}

Neovasculature distributed inside the atherosclerotic plaque is the primary route of inflammatory cell infiltration, a key process for plaque initiation, progression, and rupture [1]. Plaque neovascularization and inflammation are associated with clinical cardiovascular events caused

\footnotetext{
* Correspondence: xzhao@cardiology.washington.edu

${ }^{4}$ Division of Cardiology, University of Washington School of Medicine, 325 9th Ave, Harborview Medical Center, Seattle, WA 98104, USA Full list of author information is available at the end of the article
}

by atherosclerosis [2-4]. As a result, inflammation is an emerging target for treatment in atherosclerosis $[5,6]$. To assess and monitor the therapeutic effects of new treatments, imaging techniques are needed that detect and quantify neovascularization and inflammation in-vivo.

Dynamic Contrast-Enhanced (DCE) cardiovascular magnetic resonance (CMR) can quantify atherosclerotic angiogenesis and inflammation in-vivo by measuring the density and function of the neovasculature through kinetic modeling. Kinetic parameters, including fractional 
plasma volume $\left(v_{p}\right)$, and the transfer constant $\left(K^{\text {trans }}\right)$, have been found to be associated with histological measurements of plaque neovasculature and macrophages [7-9]. Based on this knowledge, DCE-CMR has been successfully used in assessment of the therapeutic response of the vasa vasorum in patients with atherosclerotic plaque, including a decrease in $K^{\text {trans }}$ after 1-year intensive lipid therapy [10]. Another study demonstrated the effect of pioglitazone on atheroma by using DCE-CMR in an animal model [11]. DCE-CMR was also utilized to monitor the nature progression of atherosclerotic plaque [12].

For such studies, reproducibility of quantitative parameters is key to study planning. However, the reproducibility of the $K^{\text {trans }}$ and $v_{p}$ measurements of human vessel wall remains largely unknown, especially for clinical studies involving multiple imaging centers. This study sought to investigate the inter-scan reproducibility of kinetic parameters in atherosclerotic plaque using dynamic contrastenhanced (DCE) CMR in a multi-center setting at 3T.

\section{Methods Study population}

This study was performed with institutional review board approval at each participating clinical site. Written informed consent was obtained prior to study procedures. Subjects who were enrolled in the Atherothrombosis Intervention in Metabolic syndrome with low HDL/high triglycerides: Impact on Global Health outcomes (AIM-HIGH) trial [13] were asked for willingness to participate in a MR reproducibility study. They were 45 and older, with dyslipidemia and clinically established atherosclerotic disease in coronary, cerebrovascular/carotid and/or peripheral arteries [13]. Subjects with any contraindication for MR examination or contrast injection were not included. Consented subjects underwent two carotid MR examinations with two weeks, which included a previously described DCE-CMR protocol. The present study, as well as most previous studies, used a bright-blood sequence for DCE-CMR [7-10], which does not allow assessment of near-normal arteries due to concerns of luminal signal contamination. Therefore, only subjects with distinct carotid plaques were included, defined as maximum wall thickness $>1 \mathrm{~mm}$ [14] measured on pre-contrast T1-weighted MR with a customized software (CASCADE) [15]. To ensure a multicenter design, each imaging site was restricted to a maximum of 6 subjects. From March 2009 to May 2012, of 47 subjects screened, 33 AIM-HIGH subjects were enrolled at 10 imaging sites. We also screened 21 subjects and enrolled 18 subjects with the same inclusion and exclusion criteria from 5 non-AIM-HIGH imaging sites to further increase the number of sites and subjects. In total, 51 subjects from 15 imaging sites were included.
This study was approved by the local IRB/RECs at each participating clinical site. A signed informed consent was obtained from participants.

\section{Magnetic resonance imaging}

All subjects were scanned twice within two weeks on 3T MR scanners (GE HealthCare or Philips Healthcare) to acquire carotid artery images using commercially available carotid phased-array coils (GE: 6-channel, Neocoil LCC, Pewaukee, WI, USA; Philips: 8-channel, SHCG, Shanghai, China). Each reproducibility scan was conducted on the same scanner platform as the baseline scan. The imaging protocol included an axial multi-slice 2D spoiled gradient recalled echo sequence to acquire DCE images. On GE scanners 8 contiguous slices were acquired, centered on the bifurcation of the index carotid artery to ensure the alignment between repeated scans. The acquisition parameters were: field of view: 160*160 mm, matrix 256*256, imaging resolution: $0.625 * 0.625 \mathrm{~mm}$, reconstructed image size $512 * 512$, $2 \mathrm{~mm}$ slice thickness, repetition time $117 \mathrm{~ms}$, echo time $5 \mathrm{~ms}$, flip angle $50^{\circ}$. On Philips scanners, the protocol was slightly different to ensure a similar coverage, signal-to-noise ratio (SNR) and temporal/spatial resolution: only 4 contiguous slices were acquired, also centered on the bifurcation of index side, with $3 \mathrm{~mm}$ slice thickness, repetition time $126 \mathrm{~ms}$. The index side was defined as the side with larger plaque. Coincident with the third dynamic scan in the sequence, $0.05 \mathrm{mmol} / \mathrm{kg}$ of a gadolinium-based contrast agent (Magnevist, Bayer Healthcare) was injected at a rate of $0.7 \mathrm{ml} / \mathrm{s}$ by a power injector. The contrast dose and injection rate was selected to avoid possible flow artifacts and weaken the requirement of high temporal resolution in DCE imaging [9]. Images were acquired at 18 time points separated by a repetition interval of $18 \mathrm{~s}$. To impose a T1-dependent signal on inflowing blood, a spatial saturation band was used.

\section{Image analysis}

A custom software (CASCADE) [15] was used for image analysis. The DCE-CMR images were analyzed to generate vasa vasorum $(\mathrm{V}-\mathrm{V})$ images as previously described [16]. This included registration and smoothing using the Kalman filtering registration and smoothing algorithm, extraction of the arterial input function, and calculation of $K^{\text {trans }}$ and $v_{p}$ for each pixel based on the Patlak kinetic model [17]. The resulting color-coded, parametric $\mathrm{V}-\mathrm{V}$ images show $K^{\text {trans }}$ in green and $v_{p}$ in red (Figure 1), allowing single channel (green or red) to be displayed. Next, the V-V image was outlined to define the lumen boundary (the border of high $v_{p}$ values within the lumen) and the outer wall boundary (the rim of high $K^{\text {trans }}$ values representing adventitial enhancement) [18] (Figure 1). 


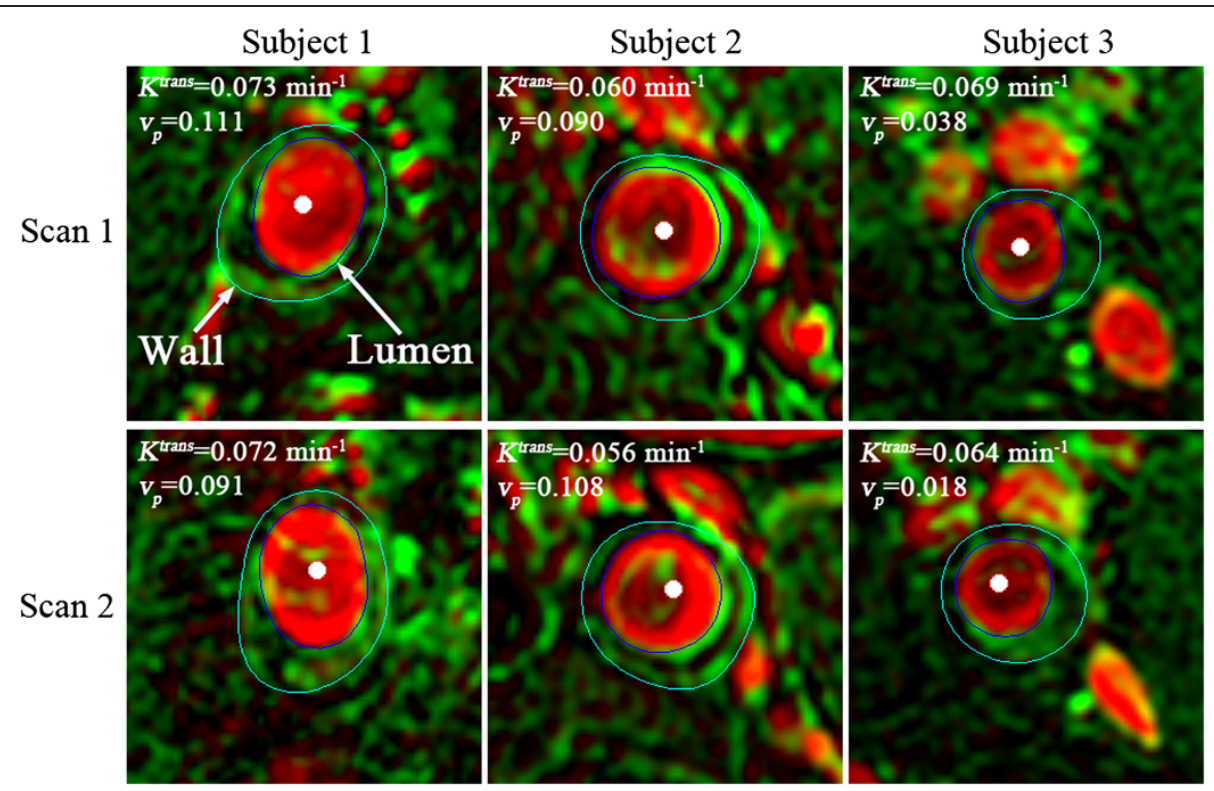

Figure 1 Representative scan-rescan V-V images (rows) of three plaques (columns). The white points indicate the carotid lumen; the white arrows point to the outlined lumen and outer wall boundaries. Green indicates $K^{\text {trans }}$ with a range from 0 to $0.2 \mathrm{~min}^{-1}$ and red indicates $v_{p}$ with a range from 0 to 1. Minor mis-resgistrations were occasionally present. The $K^{\text {tran }}$ and $v_{p}$ values shown in each figure are the measurements of the whole plaque.

Each scan was analyzed independently, blinded to the other scan and subject information. First, one trained reviewer (J. S., 3 years experiences in plaque analysis) identified all the boundaries on $\mathrm{V}-\mathrm{V}$ images after checking the protocol compliance. Then, a second reviewer (H. C., 4 years experiences in plaque analysis) performed peerreview of the boundaries defined by the primary reviewer. Cases in which there was disagreement were discussed with the primary reviewer to reach the consensus opinion.

Lastly, $K^{\text {trans }}$ and $v_{p}$ measurements were calculated by averaging all pixels within the vessel wall, except for those within $1 \mathrm{~mm}$ of the lumen contour (excluded automatically by CASCADE). This exclusion minimized any influence from the high intensity lumen signal in bright-blood DCE images due to partial volume effects, blurring, and motion.

\section{Statistical analysis}

The means of the scan 1 and scan $2 K^{\text {trans }}$ and $v_{p}$ values were compared using the paired t-test. The overall between-scan reproducibility was estimated using a linear mixed model. The reproducibility results are presented as the between-scan standard deviation (SD), the betweenscan CV (between-scan SD divided by the grand mean) [19] and the intra-class correlation (ICC). The 95\% confidence intervals for these three reproducibility statistics were calculated using the non-parametric bootstrap. The differences in means and between-scan SDs across the GE and Phillips platforms were tested using the linear mixed model and the permutation test, respectively.
To explore the association of each subject's betweenscan reproducibility with their plaque area we plotted the subjects' means and plaque sizes (the smaller of the two scans' plaque areas) against the subjects' betweenscan SD. The association between the two factors and the between-scan SD was tested using the Spearman correlation $(\rho)$ test. The least-squares fit was used to highlight the trends in the scatter plots.

For each kinetic measurement, the $\mathrm{CV}$ estimate was used to calculate sample sizes needed to detect differences in\% changes (follow-up minus baseline, normalized by the baseline mean) between two treatment arms. The sample size calculations used the two-sided unpaired t-test with $80 \%$ power and the 0.05 significance level [20] without considering the exclusion rate. The $\mathrm{CV}$ for the change (follow-up minus baseline) was calculated from the $\mathrm{CV}$ for a single time measurement (obtained in the reproducibility analysis) by multiplying it by $\sqrt{ } 2$. This calculation assumes that the measurement error is the primary source of variability within each treatment group (most importantly that the expected treatment effect does not differ across patients).

The calculations were carried out in R (The R Foundation for Statistical Computing, Vienna, Austria), version 2.15.2. A p-value less than 0.05 was used to denote statistical significance.

\section{Results}

Of the 51 subjects included in this study, 10 (19.6\%) were excluded due to any significant violation of the 
DCE imaging protocol in at least one scan, including incorrect time interval $(n=3)$, too few time frames $(n=2)$, failure to inject contrast agent $(n=2)$ and improper alignment of images $(n=3)$. Additionally, $6(11.8 \%)$ subjects were excluded for uninterpretable image quality in at least one scan, generally due to severe subject motion and/or low SNR. The remaining 35 (68.6\%) subjects were available for reproducibility assessment. These subjects were from 14 sites with $2.5 \pm 1.5$ (mean \pm standard deviation) per site range (1 to 6). Demographic and clinical characteristics of these subjects are given in Table 1.

DCE-CMR protocol non-compliance and image quality issues were widely distributed across imaging sites. The 10 instances of protocol non-compliance were spread among 8 imaging sites and the 6 instances of poor image quality were spread among 5 sites. No site accounted for more than 2 exclusions due to protocol non-compliance and image quality combined. Only in one site, all the scanned subjects ( 2 subjects) were not included in the analysis due to protocol non-compliance.

Figure 1 shows representative examples of the matched $\mathrm{V}-\mathrm{V}$ images for two scans of three carotid arteries, showing $K^{\text {trans }}$ in green and $v_{p}$ in red. Mean $K^{\text {trans }}$ and $v_{p}$ for the first and second scan are compared in Table 2. Figure 2a and $\mathrm{b}$ show the Bland-Altman plots of $K^{\text {trans }}$ and $v_{p}$, respectively.

Overall reproducibility parameters for $K^{\text {trans }}$, and $v_{p}$ are shown in Table 3. For $K^{\text {trans }}$, the CV was $25 \%(95 \%$ CI 15\%-28\%) and the ICC was 0.65 (95\% CI 0.57-0.85) suggesting moderate reproducibility of that parameter. The parameter $v_{p}$, on the other hand, exhibited substantially worse reproducibility with a CV of $62 \%$ (95\% CI $36 \%-73 \%)$ and ICC of 0.28 (95\% CI 0.14-0.70). The within-subject SD was not significantly related to the mean value for both $K^{\text {trans }}(\rho=-0.02, p=0.9$, Figure $2 c)$ and $v_{p}(\rho=0.25, \mathrm{p}=0.15$, Figure $2 \mathrm{~d})$.

The poor performance of $v_{p}$ had a significant association with plaque size exhibiting a negative Spearman correlation $(\rho=-0.45, p=0.007$; Figure $2 f)$. If subjects

Table 1 Demographic and clinical characteristics in $\mathbf{3 5}$ subjects included in reproducibility study

\begin{tabular}{ll}
\hline & mean \pm SD (range) or N (\%) \\
\hline Age (years) & $61 \pm 8(45-79)$ \\
Gender (female) & $12(34 \%)$ \\
History of Myocardial Infarction & $9(26 \%)$ \\
History of Stroke & $3(9 \%)$ \\
History of Hypertension & $28(80 \%)$ \\
History of Diabetes & $10(29 \%)$ \\
LDL-C (mg/dl) & $81 \pm 34(33-205)$ \\
Triglycerides (mg/dl) & $163 \pm 57(80-349)$ \\
HDL-C, (mg/dl) & $35.3 \pm 6.1(20.0-49.8)$ \\
\hline
\end{tabular}

with analyzed areas smaller than $25 \mathrm{~mm}^{2}$ were excluded, leaving 19 subjects for analysis, the CV and ICC for $v_{p}$ improved to $28 \%$ (95\% CI 18\%-31\%) and 0.73 (0.67-0.89), respectively. The association between $K^{\text {trans }}$ reproducibility and plaque size showed only marginal significance $(\rho=-0.33, p=0.06$; Figure $2 \mathrm{e})$. Little change in the ICC and $\mathrm{CV}$ of $K^{\text {trans }}$ was observed for the $25 \mathrm{~mm}^{2}$ cutoff.

Based on the reproducibility results above, the sample sizes needed to detect differences in change of each kinetic parameter between two treatment arms were computed without considering the exclusion rate (Figure 3). For $\mathrm{K}^{\text {trans }}, 197,50$ and 23 subjects per arm would be needed to detect differences of $10 \%, 20 \%$ and $30 \%$, respectively. For $v_{p}, 1224,307$ and 137 subjects per arm would be needed, respectively. The required sample sizes for $v_{p}$ reduced to 254, 64, and 29 if subjects with analyzed plaque areas less than $25 \mathrm{~mm}^{2}$ were excluded.

The use of 2 primary scanner platforms (GE and Philips) allowed us to also evaluate whether these findings depended on vendor. In total, 20 subjects scanned on GE scanners were included in the study and 15 subjects scanned on Philips scanners were included. As summarized in Table 3, the differences in means, standard deviations and $\mathrm{CVs}$ of kinetic parameters across platforms were relatively small and not statistically significant.

\section{Discussion}

This study provides an evaluation of scan-rescan reproducibility of kinetic parameters $\left(K^{\text {trans }}\right.$ and $\left.v_{p}\right)$ generated from DCE-CMR using 3T MR scanners for multi-center studies of carotid atherosclerosis. We found that $K^{\text {trans }}$ had moderate but acceptable reproducibility in the multi-center setting, with an overall CV of $25 \%$. For this level of reproducibility, a $20 \%$ greater relative reduction in $K^{\text {trans }}$ could be detected with $80 \%$ power and $\mathrm{p}=0.05$ using 50 subjects per arm. Notably, a single arm study involving 28 subjects on lipid lowering therapy using similar techniques reported slightly greater than $20 \%$ reduction in $K^{\text {trans }}$ over a period of 1 year [10]. As a comparison for the reproducibility of vessel wall inflammation quantification, a widely used serum-based inflammation biomarker, high-sensitive C-Reactive Protein, showed a large test-retest variation with $\mathrm{CV}$ of $46.2 \%$ [21].

For $v_{p}$, on the other hand, the reproducibility was lower, with an overall $\mathrm{CV}$ of $62 \%$, indicating it is not a preferable

Table 2 Comparison of kinetic parameters across scans

\begin{tabular}{llll}
\hline & \multicolumn{2}{l}{ Mean (Standard deviation) } & \\
\cline { 2 - 3 } & Scan 1 & Scan 2 & $\mathbf{p}^{\ddagger}$ \\
\hline$K^{\text {trans* }}$ & $0.061(0.022)$ & $0.063(0.030)$ & 0.6 \\
$V_{p}^{+}$ & $0.064(0.053)$ & $0.070(0.046)$ & 0.5 \\
\hline
\end{tabular}

$K^{\text {trans: }}$ : transfer constant in $\min ^{-1}$.

${ }^{\dagger} v_{p}$ : plasma volume fraction (unitless).

Fpaired t-test. 

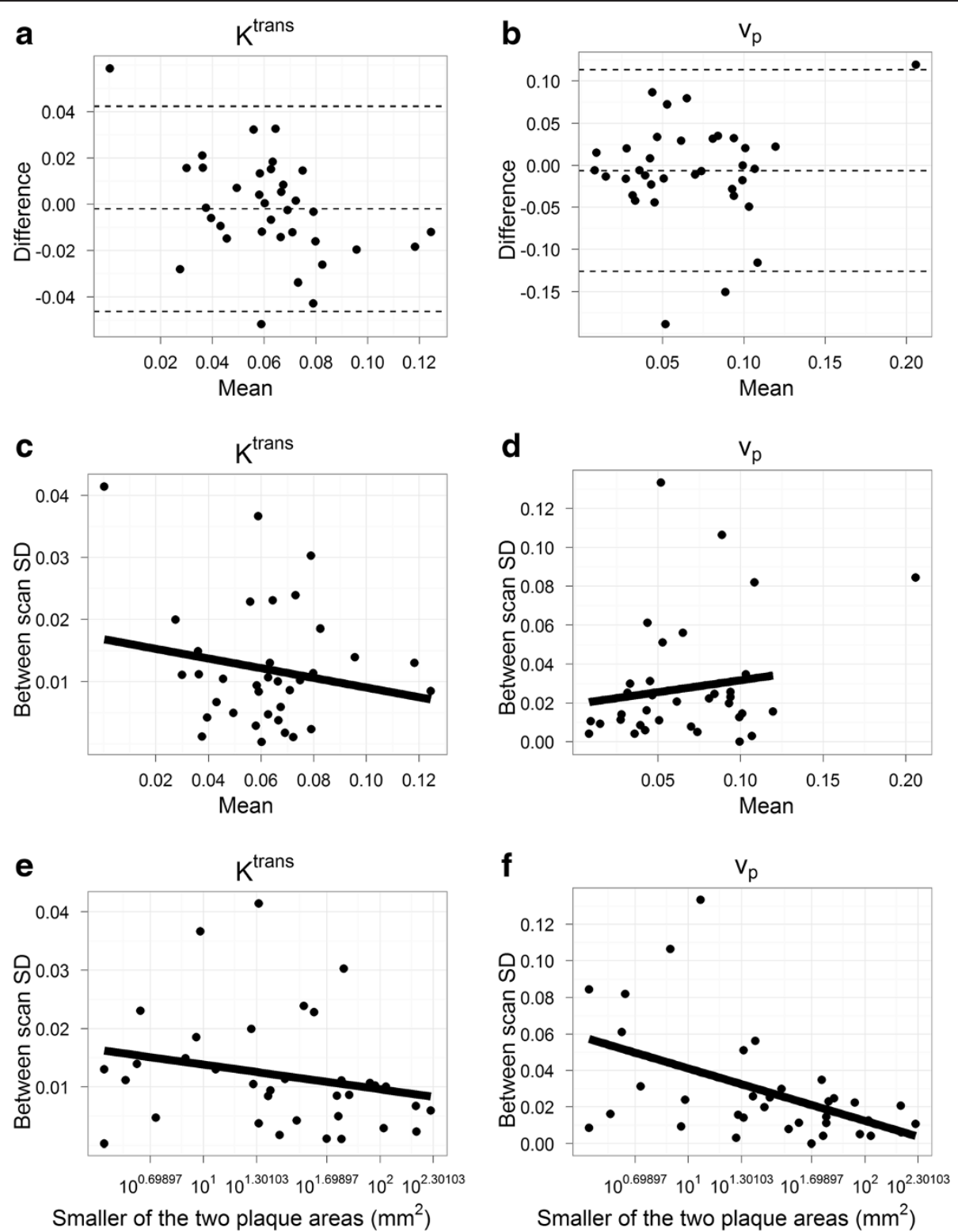

Figure 2 Relationship of reproducibility with the mean measurement and with the plaque area. $\mathbf{a}$. Bland-Altman plot of $K^{\text {trans }}$ b. Bland-Altman plot of $v_{p}$. c. Per-subject mean $K^{\text {trans }}\left(\min ^{-1}\right)$ vs. per-subject between-scan SD of $K^{\text {trans }}(\rho=-0.02, p=0.9)$, d. Per-subject mean $v_{p}$ (unitless) vs. per-subject between-scan SD of $v_{p}(\rho=0.25, p=0.15)$. e. Plaque areas (on the log-scale) vs. per-subject between-scan SD of $K^{\text {trans }}$ $(\rho=-0.33, p=0.06)$, f. Plaque areas (on the log-scale) vs. per-subject between-scan SD of $v_{p}(\rho=-0.45, p=0.007)$. Dashed lines in panel (a) and (b) indicate the mean differences and limits of agreement ( $\pm 2 \times$ SD of differences) for Bland-Altman plots. Solid lines in panels (d-f) are least square fits. Fitted line in panel (d) excludes one outlier with mean $v_{p}$ above 0.20 .

parameter for clinical studies. The reason may be that $v_{p}$, which represents tissue blood supply, is more vulnerable to signal influences from lumen. The much higher reproducibility, as shown in a subset of patients with larger plaques, supports this hypothesis. Notably, previous histological validation studies [7-9] were all on patients with large lesions, further indicating $v_{p}$ is more reliable parameter in large plaques. Additionally, the limited temporal resolution may also introduce some variance for $v_{p}$ [22]. As such, future studies involving limiting $v_{p}$ are suggested to use subjects with larger lesions.

Of note, the reproducibility of DCE-CMR parameters found in this multi-center experience is similar to that reported in a recent single-center study [9]. In that study, reported ICCs for $K^{\text {trans }}$ and $v_{p}$ were 0.79 and 0.48 , respectively. CVs were $16 \%$ and $26 \%$. The somewhat better reproducibility in that study may reflect the use of a single imaging center. In addition, their population included only subjects with at least 30\% carotid 
Table 3 Grand means, between-scan standard deviations (SDs) and coefficients of variation for kinetic parameters by vendor

\begin{tabular}{llll}
\hline & Mean & SD & CV (\%) $\left(\mathbf{9 5} \% \mathbf{C l}^{\ddagger}\right)$ \\
\hline$K^{\text {trans* }}$ & & & \\
All & 0.062 & 0.015 & $25 \%(15 \%-28 \%)$ \\
GE & 0.064 & 0.017 & $27 \%(16 \%-40 \%)$ \\
Philips & 0.060 & 0.013 & $21 \%(10-22 \%)$ \\
$p^{\S}$ & 0.6 & 0.12 & 0.12 \\
$V_{p}^{+}$ & & & \\
All & 0.067 & 0.042 & $62 \%(36 \%-73 \%)$ \\
GE & 0.068 & 0.042 & $62 \%(46-73 \%)$ \\
Philips & 0.066 & 0.040 & $60 \%(18-71 \%)$ \\
$p^{\S}$ & 0.8 & 0.9 & 0.9 \\
\hline
\end{tabular}

${ }^{*} K^{\text {trans }}$ : transfer constant in $\mathrm{min}^{-1}$.

${ }^{\dagger} v_{p}$ : plasma volume fraction (unitless).

${ }^{\ddagger} \mathrm{Cl}$ : confidence interval.

${ }^{s}$ the linear mixed model test for the comparison of the means and the permutation test for the comparison of the SDs and CVs (NS : $p>0.05$ ).

stenosis, which may have enriched their study with larger, more reproducible lesions.

Another key finding of this study involved the use of both GE and Philips scanner platforms. Although each subject was scanned on the same scanner, the measurements and variability could have been platform dependent, because the two platforms required slightly different imaging protocols and coils. We found, however, no significant differences in mean values or reproducibility metrics when these two platforms were compared. This implies that multi-center studies may not have to be restricted to a single imaging platform.

To our best knowledge, this study is the first to study the implementation of DCE-CMR in a multi-center setting. We found that significant protocol violation in any of the paired scans could affect as high as $20 \%$ of cases, such as using incorrect time interval, insufficient time frames, improper positioning of imaging slabs, and failure to inject contrast agent. Other than that, around $12 \%$ subjects were excluded because of poor imaging quality. In this study, because sites were limited to no more than 6 subjects, inexperience was likely a factor in non-compliance. Moreover, the high rate of poor image quality indicates that local operators have difficulties to determine whether the scanned images have adequate quality for quantitative analysis, because DCE-CMR needs customized post-processing and modeling. For future DCE-CMR studies, the following improvements are needed to reduce the dropout rate: 1) optimization of the experience of MR technologists performing the scans, including more intensive hands-on training, mentoring on subject instruction and provision of timely, ongoing feedback for quality control; 2) technological advancements including development of more rapid DCE-CMR

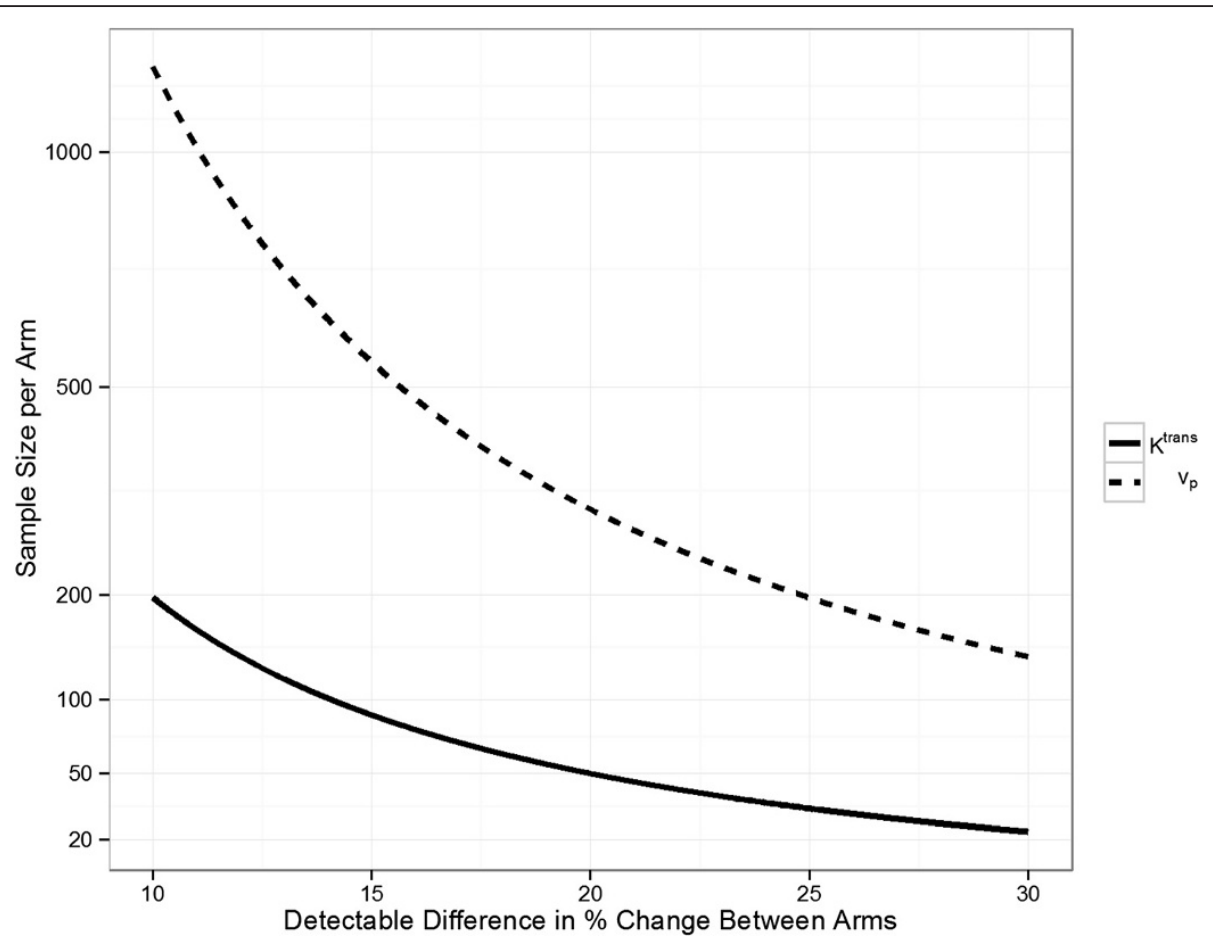

Figure 3 Sample size analysis. Sample size needed for each kinetic parameter to detect a difference in\% change between treatment arms with $80 \%$ power using a two-sided unpaired t-test at the 0.05 significance level. The solid and dotted lines are the required sample sizes to detect changes from $10 \%$ to $30 \%$ for $K^{\text {trans }}$ and $v_{p}$, respectively. 
protocols, implementation of motion correction reconstruction methods, and improvement in imaging coil design for deep carotid arteries; and 3) strict quality control measures [23], including timely assessment of image quality and imaging protocol adherence at the central review site after post-processing, recall of subjects with poor image quality or protocol violation for repeat scan.

The source of the observed measurement variances may relate to several factors that could also be addressed for further improvement. First, patient positioning differences and subsequent image misregistration between the two scans (Figure 1), even among different time points in DCE series, may be an important factor, because the imaging slice thickness $(2 \mathrm{~mm})$ is much larger than the in-plane resolution $(0.625 \mathrm{~mm})$. Second, the relatively low temporal resolution (18 s) can introduce variance into the arterial input function extraction. However, the specified spatial and temporal resolution used in this study is a tradeoff with the SNR required to obtain adequate image quality for DCE-CMR analysis. Patient movement during DCE scan can cause blurring and artifacts in images that affect the kinetic analysis. Coil placement differences also can introduce variance. Finally, there may be some natural variability between repeated scans due to perfusion regulation.

One technical limitation of this study is that current analysis of bright-blood DCE images excluded a $1 \mathrm{~mm}$ layer near the lumen boundary for measuring mean $K^{\text {trans }}$ and $v_{p}$, precluding the evaluation of thinner vessel walls. However, this is a tradeoff to minimize the signal contamination in the vessel wall from the high-intensity lumen, which is a major difficulty in bright-blood DCE imaging. Thus, studies using bright-blood DCE techniques are more applicable to larger, more advanced lesions other than small early lesions. Recently proposed black-blood DCE imaging technique may be a solution for thin vessel walls $[11,12,24]$. This study was prospectively designed to estimate scan-rescan measurement errors that have potential utility for future serial imaging studies. Image analysis followed the conventional way in clinical trials where two readers analyze each case sequentially to reach consensus opinion before measurement is locked to avoid human errors and biases. Thus, intra- and inter-reader reproducibility was not assessed. Compared to inter-scan variability, reader variability may be a less problem given that most post-processing steps of DCECMR analysis are fully automated. Nonetheless, these metrics need to be studied in future investigations before the technique is used in the clinical setting.

\section{Conclusions}

This study is the first to establish scan-rescan reproducibility and measurement variance of DCE-CMR of carotid atherosclerosis in a multi-center, multi-platform setting. We found both $K^{\text {trans }}$ and $v_{p}$ derived from DCE-CMR, have moderate reproducibility, the latter with lesions that have a minimum analyzed area greater than $25 \mathrm{~mm}^{2}$. Based on these findings, changes in $K^{\text {trans }}$ would be detectable in a prospective study with relatively modest sample size, indicating that it is feasible to run multi-center trials using vessel wall DCE-CMR. However, the rate of uninterruptable images in this study highlights the need for intensive operator training, optimized imaging protocols timely, and ongoing quality control, in future multicenter studies.

\section{Abbreviations \\ AIM-HIGH: Atherothrombosis Intervention in Metabolic syndrome with low HDL/high triglycerides: Impact on Global Health outcomes trial; DCE: Dynamic Contrast-Enhanced; CMR: Cardiovascular Magnetic Resonance; ICC: Intra-Class Correlation coefficient; CV: Coefficient of Variation; SD: Standard Deviation; $K^{\text {rrans: }}$ Transfer constant; $v_{p}$ : Plasma volume; SNR: Signal-to-Noise Ratio.}

\section{Competing interests}

WSK is a former employee of VPDiagnostics, Inc.

\section{Authors' contributions}

$\mathrm{HC}$ carried out the design of the study, data analysis, data interpretation, and drafted the manuscript. JS participated in study design, data analysis and interpretation, and helped to draft the manuscript; WSK conceived of the study, participated in its design and data interpretation, and helped to draft the manuscript; NB participated in the data interpretation and manuscript revision; MBN performed the statistical analysis, participated the data interpretation and manuscript revision; DSH helped to carry out the statistical analysis, participated in the data interpretation and manuscript revision; DI helped in data acquisition, participated in the data interpretation and manuscript revision; YX participated in the data analysis and manuscript revision; KY participated in the data analysis and manuscript revision; SP participated in the data interpretation and manuscript revision; $\mathrm{CY}$ conceived of the study, participated in the data interpretation and manuscript revision; KDO conceived of the study, participated in the study design, data interpretation and manuscript revision; XQZ conceived of the study, participated in the study design, data interpretation, and manuscript revision; All authors read and approved the final manuscript and the submission.

\section{Acknowledgements}

Funding provided by a research grant from the Foundation for the $\mathrm{NIH}$ Biomarkers Consortium made possible by funds from Merck, Pfizer, and Abbott and by R01HL088214 and R01HL089504. We would like to thank the Biomarkers Consortium at Foundation of the $\mathrm{NIH}$. Project team members and their affiliation are the followings:

(1) Patrice Desvigne-Nickens, NHLBI

(2) Jerome Fleg, NHLBI

(3) Michael Klimas, Merck

(4) Elizabeth Stafford, CDRH/FDA

(5) Robert J Padley, Abbott

(6) Brad Wyman, Pfizer

(7) Chun Yuan, University of Washington

(8) Xue-Qiao Zhao, University of Washington

(9) Jessica Ratay, FNIH

(10) Maria Vassileva, FNIH

We appreciate support from the AIM-HIGH Study, Principal Investigators: B. Gregory Brown, MD, PhD (retired); Jeffrey L. Probstfield, MD; William E. Boden, MD.

We would like to thank the investigators who were instrumental in recruiting subjects and obtaining the MR images at the 10 AIM-HIGH and 6 non-AIMHIGH sites listed below:

(1) Cardiovascular Consultants and Barrows Neurological Institute: Chris Geohas and Jim Pipe.

(2) Mayo Clinic: Stephen Kopecky and John Huston 
(3) University of Southern California and Long Beach VA: Patrick Colletti and Moti Keshyap

(4) University of Calgary: Todd Anderson and Richard Frayne

(5) University of Washington: Xue-Qiao Zhao, Kevin D. O'Brien, Chun Yuan and Thomas Hatsukami

(6) Wake Forest University and Duke University: J. Robin Crouse, David Herrington and John R. Guyton

(7) Lawson Health Research Institute and Robarts Research Institute: William

J. Kostuk and Brian Rutt

(8) Methodist Hospital and Baylor School of Medicine: Alan Hoffman, Peter Jones and Joel Morrisett

(9) Vancouver General Hospital and University of British Columbia: Anthony Fung and Alex Mackay

(10) University of Maryland and Johns Hopkins University: Michael Miller and Bruce Wasserman

(11) Beijing Friendship Hospital and Beijing Hospital: Shumei Zhao and Cheng Zhou

(12) Beijing Union Medical College and Tsinghua University: Hua Bai, Feiyu Li and Xihai Zhao

(13) Shanghai Renji Hospital: Xiaosheng Liu and Jianrong Xu

(14) Oregon Health Sciences University: Jerzy Szumowski

(15) Michigan State University: Kevin DeMarco

(16) UT Southwestern: Jarett Berry

We would like to thank GE Healthcare and Philips Healthcare for providing imaging coil for this study.

\section{Author details}

${ }^{1}$ Department of Biomedical Engineering, Center for Biomedical Imaging Research, School of Medicine, Tsinghua University, Beijing 100084, China. ${ }^{2}$ Department of Radiology, University of Washington, 850 Republican St, Seattle, WA 98109, USA. ${ }^{3}$ The Mountain-Whisper-Light Statistics, 1827 23rd Ave. East, Seattle, WA 98112, USA. ${ }^{4}$ Division of Cardiology, University of Washington School of Medicine, 325 9th Ave, Harborview Medical Center, Seattle, WA 98104, USA.

Received: 14 February 2014 Accepted: 30 June 2014

Published: 1 August 2014

\section{References}

1. Libby P. Inflammation in atherosclerosis. Nature. 2002; 420(6917):868-74.

2. Mofidi R, Crotty TB, McCarthy P, Sheehan SJ, Mehigan D, Keaveny TV. Association between plaque instability, angiogenesis and symptomatic carotid occlusive disease. Br J Surg. 2001; 88(7):945-50.

3. McCarthy MJ, Loftus IM, Thompson MM, Jones L, London NJ, Bell PR, Naylor AR, Brindle NP. Angiogenesis and the atherosclerotic carotid plaque: an association between symptomatology and plaque morphology. J Vasc Surg. 1999; 30(2):261-8.

4. Dunmore BJ, McCarthy MJ, Naylor AR, Brindle NP. Carotid plaque instability and ischemic symptoms are linked to immaturity of microvessels within plaques. J Vasc Surg. 2007; 45(1):155-9.

5. Charo IF, Taub R. Anti-inflammatory therapeutics for the treatment of atherosclerosis. Nat Rev Drug Discov. 2011; 10(5):365-76.

6. Weber $\mathrm{C}$, Noels $\mathrm{H}$. Atherosclerosis: current pathogenesis and therapeutic options. Nat Med. 2011; 17(11):1410-22.

7. Kerwin W, Hooker A, Spilker M, Vicini P, Ferguson M, Hatsukami T, Yuan C. Quantitative magnetic resonance imaging analysis of neovasculature volume in carotid atherosclerotic plaque. Circulation. 2003; 107(6):851-6.

8. Kerwin W, O'Brien K, Ferguson M, Polissar N, Hatsukami T, Yuan C. Inflammation in carotid atherosclerotic plaque: a dynamic contrastenhanced MR imaging study. Radiology. 2006; 241(2):459-68.

9. Gaens ME, Backes WH, Rozel S, Lipperts M, Sanders SN, Jaspers K, Cleutjens JP, Sluimer JC, Heeneman S, Daemen MJ, Welten RJ, Daemen JW, Wildberger JE, Kwee RM, Kooi ME. Dynamic contrast-enhanced MR imaging of carotid atherosclerotic plaque: model selection, reproducibility, and validation. Radiology. 2013; 266(1):271-9.

10. Dong L, Kerwin WS, Chen H, Chu B, Underhill HR, Neradilek MB, Hatsukami TS, Yuan C, Zhao XQ. Carotid artery atherosclerosis: effect of intensive lipid therapy on the vasa vasorum-evaluation by using dynamic contrast-enhanced MR imaging. Radiology. 2011; 260(1):224-31.

11. Vucic E, Dickson SD, Calcagno C, Rudd JH, Moshier E, Hayashi K, Mounessa JS, Roytman M, Moon MJ, Lin J, Tsimikas S, Fisher EA, Nicolay K, Fuster V,
Fayad ZA. Pioglitazone modulates vascular inflammation in atherosclerotic rabbits noninvasive assessment with FDG-PET-CT and dynamic contrast-enhanced MR imaging. JACC Cardiovasc Imaging. 2011; 4(10):1100-9.

12. Chen H, Ricks J, Rosenfeld M, Kerwin WS. Progression of experimental lesions of atherosclerosis: assessment by kinetic modeling of black-blood dynamic contrast-enhanced MRI. Magn Reson Med. 2013; 69(6):1712-20.

13. Boden WE, Probstfield $J$, Anderson T, Chaitman BR, Desvignes-Nickens $P$, Koprowicz K, McBride R, Teo K, Weintraub W, Investigators A-H. Niacin in patients with low HDL cholesterol levels receiving intensive statin therapy. N Engl J Med. 2011; 365(24):2255-67.

14. Wang J, Liu H, Sun J, Xue H, Xie L, Yu S, Liang C, Han X, Guan Z, Wei L, Yuan C, Zhao X, Chen H. Varying Correlation Between 18FFluorodeoxyglucose Positron Emission Tomography and Dynamic Contrast-Enhanced MRI in Carotid Atherosclerosis: Implications for Plaque Inflammation. Stroke. 2014; 45(6):1842-5.

15. Kerwin W, Xu D, Liu F, Saam T, Underhill H, Takaya N, Chu B, Hatsukami T, Yuan C. Magnetic resonance imaging of carotid atherosclerosis: plaque analysis. Top Magn Reson Imaging. 2007; 18(5):371-8.

16. Kerwin W, Oikawa M, Yuan C, Jarvik G, Hatsukami T. MR imaging of adventitial vasa vasorum in carotid atherosclerosis. Magn Reson Med. 2008; 59(3):507-14.

17. Patlak C, Blasberg R, Fenstermacher J. Graphical evaluation of blood-tobrain transfer constants from multiple-time uptake data. J Cereb Blood Flow Metab. 1983; 3(1):1-7.

18. Sun J, Song Y, Chen H, Kerwin WS, Hippe DS, Dong L, Chen M, Zhou C, Hatsukami TS, Yuan C. Adventitial perfusion and intraplaque hemorrhage: a dynamic contrast-enhanced MRI study in the carotid artery. Stroke. 2013; 44(4):1031-6.

19. Grothues F, Smith GC, Moon JC, Bellenger NG, Collins P, Klein HU, Pennell DJ. Comparison of interstudy reproducibility of cardiovascular magnetic resonance with two-dimensional echocardiography in normal subjects and in patients with heart failure or left ventricular hypertrophy. Am J Cardiol. 2002; 90(1):29-34.

20. Saam T, Kerwin WS, Chu B, Cai J, Kampschulte A, Hatsukami TS, Zhao XQ, Polissar NL, Neradilek B, Yarnykh VL, Flemming K, Huston J III, Insull W Jr, Morrisett JD, Rand SD, DeMarco KJ, Yuan C. Sample size calculation for clinical trials using magnetic resonance imaging for the quantitative assessment of carotid atherosclerosis. J Cardiovasc Magn Reson. 2005; 7(5):799-808.

21. Morton G, Schuster A, Jogiya R, Kutty S, Beerbaum P, Nagel E. Inter-study reproducibility of cardiovascular magnetic resonance myocardial feature tracking. J Cardiovasc Magn Reson. 2012; 14:43

22. Chen H, Li F, Zhao X, Yuan C, Rutt B, Kerwin WS. Extended graphical model for analysis of dynamic contrast-enhanced MRI. Magn Reson Med 2011; 66(3):868-78

23. Boussel L, Arora S, Rapp J, Rutt B, Huston J, Parker D, Yuan C, Bassiouny H, Saloner D, Investigators M. Atherosclerotic plaque progression in carotid arteries: monitoring with high-spatial-resolution MR imaging-multicenter trial. Radiology. 2009; 252(3):789-96

24. Calcagno C, Cornily J, Hyafil F, Rudd J, Briley-Saebo K, Mani V, Goldschlager $G$, Machac J, Fuster V, Fayad Z. Detection of neovessels in atherosclerotic plaques of rabbits using dynamic contrast enhanced MRI and 18 F-FDG PET. Arterioscler Thromb Vasc Biol. 2008; 28(7):1311-7.

doi:10.1186/s12968-014-0051-7

Cite this article as: Chen et al: Scan-rescan reproducibility of quantitative assessment of inflammatory carotid atherosclerotic plaque using dynamic contrast-enhanced 3T CMR in a multi-center study. Journal of Cardiovascular Magnetic Resonance 2014 16:51. 